
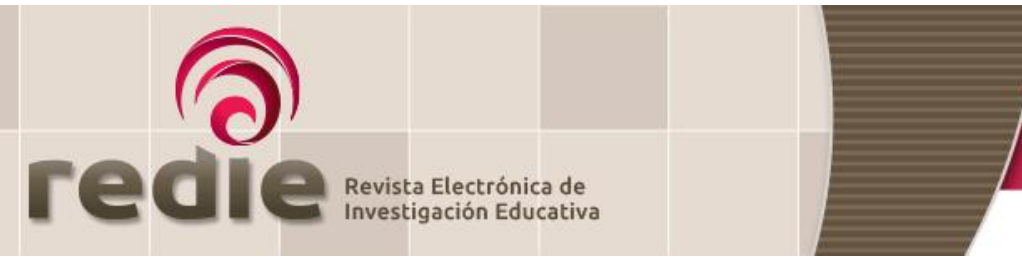

Vol. 21, 2019/e21

\title{
¿Qué piensan los futuros maestros sobre la disposición y la seguridad para enseñar Matemáticas? Algunas propuestas para la formación inicial
}

\section{What do Pre-Service Teachers Think about the Ability and Confidence Needed to Teach Maths? Some Proposals for Initial Training}

Ángel Alsina (*) angel.alsina@udg.edu

Paula López Serentill (*) paula.lopez@udg.edu

(*) Universidad de Girona

(Recibido: 13 de marzo de 2017; Aceptado para su publicación: 5 de octubre de 2017)

Cómo citar: Alsina, A. y López, P. (2019). ¿Qué piensan los futuros maestros sobre la disposición y la seguridad para enseñar matemáticas? Algunas propuestas para la formación inicial. Revista Electrónica de Investigación Educativa, 21, e21, 1-11. doi.10.24320/redie.2019.21.e21.1867

\section{Resumen}

En este estudio se analizan las creencias de 141 futuros maestros sobre la disposición y la seguridad para enseñar matemáticas. Para la identificación de estas creencias se han administrado dos cuestionarios previamente validados y se han analizado los datos a través de una metodología cuantitativa. Los resultados muestran que más de la mitad de los estudiantes creen que no todas las personas tienen la misma capacidad para las matemáticas; un porcentaje significativo se muestra más inseguro para enseñar matemáticas que otras disciplinas; y los estudiantes que presentan creencias más positivas proceden de un bachillerato científico. Estos resultados aportan información para diseñar estrategias didácticas durante la formación inicial que favorezcan el desarrollo profesional de los futuros maestros.

Palabras clave: Enseñanza de las matemáticas, formación del profesorado, formación inicial, habilidad pedagógica.

\section{Abstract}

This study analyzes the beliefs of 141 pre-service teachers on the ability and confidence needed to teach mathematics. To identify these beliefs, two previously validated questionnaires were administered and data was analyzed using a quantitative methodology. The results show that over half of students believe that not everybody has the same mathematical ability, a significant percentage are less confident teaching mathematics than other subjects, and students who exhibited more positive beliefs studied a high school program with a concentration in science. The results provide information that can be used to design didactic strategies that promote pre-service teachers' professional development during their initial training.

Keywords: Mathematics education, teacher training, basic training, teaching skills. 


\section{Introducción}

Los futuros maestros acceden a los estudios universitarios habiendo establecido diferentes relaciones con las asignaturas, como por ejemplo el desinterés, la apatía, la ansiedad e incluso el temor hacia al contenido y su aprendizaje, o en el lado opuesto, el interés, el anhelo, la tranquilidad o la seguridad, entre otras. Estas relaciones están determinadas por factores como el autoconcepto, la aptitud, el papel que ejerce el profesor, etc. (Beauchamp y Thomas, 2009).

En el caso concreto de las matemáticas, algunos estudios preliminares han revelado el sentimiento de inferioridad de los estudiantes para maestro ante esta materia (Caballero, Blanco y Guerrero, 2008; Op 't Eynde, De Corte y Verschaffel, 2002; Ministerio de Educación, Cultura y Deporte, 2012; López y Alsina, 2016). Gómez-Chacón (2000) indica que sería un error atribuir esta visión sólo a un asunto intelectual, puesto que el dominio afectivo también juega un papel importante.

McLeod (1992) distinguió tres constructos dentro del dominio afectivo: emociones, actitudes y creencias. DeBellis y Goldin (1999) añadieron los valores como un cuarto constructo, y unos años después Hannula, Op 't Eynde, Schlöglmann y Wedege (2007) propusieron un modelo sobre el dominio afectivo en educación matemática que, además de los constructos anteriores, considera también el contexto sociocultural y del aula.

En relación con las creencias, que es el aspecto en el que nos interesa profundizar, asumimos la acepción de Gómez-Chacón, Op 't Eynde y De Corte (2006), según la cual una creencia nunca se sostiene con independencia de otras, por ello se suele hablar de sistemas de creencias y no de creencias aisladas.

Este sistema de creencias, como se ha indicado, se va configurando durante la formación pre-universitaria a partir de las relaciones que los estudiantes establecen con las matemáticas. Di Martino y Zan (2010) establecen tres dimensiones interconectadas a partir de un estudio con más de 1,600 estudiantes de Primaria a Bachillerato: la disposición emocional hacia las matemáticas (positiva-negativa); b) la visión de las matemáticas (relacional-instrumental); y c) la competencia matemática percibida (alta-baja). Estos autores concluyen que el profesorado debería aprender a tratar estas dimensiones, por lo que subrayan la importancia de incorporarlas como parte del conocimiento didáctico durante la formación de maestros. En este sentido, exponen que:

La reflexión sobre el papel del maestro en las relaciones de los estudiantes con las matemáticas conduce a la necesidad de reflexionar con los futuros maestros acerca de su visión de las matemáticas y sus propias teorías sobre el éxito. (Di Martino y Zan, 2010, p. 46).

Otras investigaciones centradas específicamente en las creencias de los futuros maestros acerca de las matemáticas y su enseñanza concluyen que están formadas por un sistema integrado que incluye las creencias sobre las matemáticas como disciplina, las creencias sobre uno mismo como aprendiz de matemáticas y las creencias sobre el funcionamiento de la clase (Caballero et al., 2008; Ministerio de Educación, Cultura y Deporte, 2012; Op 't Eynde et al., 2002). Los principales resultados de estos estudios preliminares coinciden en que los futuros maestros tienden a percibir las matemáticas como una asignatura compleja cuyo aprendizaje depende en gran medida de la capacidad innata, junto con el papel que ejerce el profesor. En este sentido, la metodología de enseñanza y la evaluación son determinantes.

Los estudios mencionados indican que los aprendices tienden a percibir tanto la enseñanza como la evaluación de las matemáticas de forma tradicional, principalmente mediante actividades basadas en la repetición y la práctica de ejercicios y la realización de exámenes. Sin embargo, las directrices contemporáneas (National Council of Teachers of Mathematics [NCTM], 2015) señalan que es necesaria una enseñanza eficaz que involucre a los estudiantes en un aprendizaje significativo mediante experiencias individuales y colaborativas que fomenten su habilidad para dar sentido a las ideas matemáticas y para razonar de manera significativa. Y respecto a la evaluación, se indica que debería ser una parte integral de la enseñanza con el objeto de ofrecer evidencias del dominio del contenido matemático importante y de las prácticas matemáticas relevantes -incluyendo estrategias y fuentes 
documentales. Además, debería servir también para moldear la retroalimentación de los estudiantes, las decisiones de enseñanza y el mejoramiento del programa.

Kaasila, Hannula y Laine (2012) señalan que los estudios acerca de la visión de las matemáticas de los futuros maestros tienen un papel importante porque revelan cómo van construyendo sus creencias sobre la enseñanza de esta disciplina. Sugieren, además, que la toma de conciencia de estas creencias es el punto de partida necesario para promover procesos de cambio y de desarrollo en el profesorado. Pero no hay que olvidar, de acuerdo con Gellert, Espinoza y Barbé (2013), que durante esta toma de conciencia pueden surgir resistencias y subversiones si el aprendiz considera que el ajuste o el cambio son imposibles o no deseados.

En este contexto, el objetivo de nuestro estudio es analizar las creencias de los futuros maestros hacia las matemáticas tanto desde la óptica de su aprendizaje como de su enseñanza, y de forma más concreta la disposición hacia la materia y la seguridad para enseñarla, con el objeto de poder comprender los sentimientos negativos y promover procesos de cambio durante la formación inicial a través de estrategias didácticas adecuadas.

\section{Método}

En la investigación han participado todos los estudiantes de primero del Grado de Maestro de la Universidad de Girona (España): 72 de Educación Infantil y 69 de Educación Primaria. Se trata, por tanto, de una muestra intencional formada por 141 futuros maestros. El estudio se realiza con estudiantes de primero para poder diseñar e implementar en los cursos posteriores estrategias que permitan mejorar las experiencias y relaciones con las matemáticas de los futuros maestros. En la tabla I se presenta la distribución de los estudiantes en función del tipo de estudios de procedencia.

Tabla I. Distribución de los estudiantes

\begin{tabular}{l|c|c|c|c|c|c}
\hline Estudios de procedencia & \multicolumn{2}{|c|}{ Grado de Infantil } & Grado de Primaria & \multicolumn{2}{c}{ Total } \\
\hline Bachillerato con matemáticas & 14 & $19.4 \%$ & 19 & $27.5 \%$ & 33 & $23.4 \%$ \\
\hline Bachillerato sin matemáticas & 14 & $19.4 \%$ & 30 & $43.5 \%$ & 44 & $31.2 \%$ \\
\hline Diplomados, licenciados & 1 & $1.4 \%$ & 3 & $4.3 \%$ & 4 & $2.8 \%$ \\
\hline $\begin{array}{l}\text { Formación Profesional (FP) } \\
\text { o Ciclo Formativo de Grado } \\
\text { Superior (CFGS) }\end{array}$ & 33 & $45.8 \%$ & 11 & $15.9 \%$ & 44 & $31.2 \%$ \\
\hline $\begin{array}{l}\text { Inicio de otros estudios } \\
\text { universitarios desde Bachillerato }\end{array}$ & 1 & $1.4 \%$ & 1 & $1.4 \%$ & 2 & $1.4 \%$ \\
\hline $\begin{array}{l}\text { Inicio de otros estudios } \\
\text { universitarios desde FP y cFGS }\end{array}$ & 7 & $9.7 \%$ & 3 & $4.3 \%$ & 10 & $7.1 \%$ \\
\hline \begin{tabular}{l} 
Mayores de 25 años \\
\hline Total
\end{tabular} & 2 & $2.8 \%$ & 2 & $2.9 \%$ & 4 & $2.8 \%$ \\
\hline
\end{tabular}

Para la obtención de datos se emplearon dos cuestionarios que fueron validados mediante juicio de expertos (López y Alsina, 2016). Estos dos cuestionarios, de acuerdo con la literatura revisada, recogen diversas creencias sobre la las matemáticas y su enseñanza (López y Alsina, 2013):

- Cuestionario I: contiene 14 preguntas cerradas politómicas que se valoran con una escala tipo Likert (1=muy en desacuerdo, 2=algo en desacuerdo, 3=ni acuerdo ni desacuerdo, 4=algo de acuerdo, 5=muy de acuerdo). Las cuatro primeras preguntas analizan las creencias acerca de la disposición y la capacidad para aprender matemáticas (ver tabla II); las siguientes cinco preguntas analizan las creencias sobre la propia aptitud para aprender matemáticas (ver tabla III); y las cinco últimas preguntas recogen datos referentes a las creencias sobre el funcionamiento de la clase (ver tabla VI).

- Cuestionario Il: contiene dos preguntas abiertas sobre las asignaturas preferidas para enseñar y el 
grado de seguridad para enseñar matemáticas, respectivamente.

Los dos cuestionarios se administraron el mismo día del primer semestre a todos los participantes durante una sesión de clase ordinaria. Para el análisis de los datos se siguieron tres fases: la primera consistió en hacer una lectura de todas las respuestas de las preguntas cerradas del Cuestionario l; en la segunda se hizo un análisis minucioso de las respuestas en las dos preguntas abiertas del Cuestionario II; y en la última se realizó un análisis cuantitativo con el programa SPSS 17. Se hizo el análisis descriptivo de las variables individuales mediante tablas de frecuencia y medidas de centralización y dispersión. Para el estudio de la relación entre variables de los dos cuestionarios se emplearon tablas de contingencia y contrastes de independencia mediante el estadístico Chi-Cuadrado, aplicando un valor del 5\% para evaluar la significación de los contrastes.

\section{Resultados}

\subsection{Análisis del sistema de creencias sobre las matemáticas y la enseñanza}

Se exponen los datos obtenidos en las 14 preguntas cerradas politómicas del Cuestionario I. Como se ha indicado, el primer grupo de preguntas recoge datos acerca de las creencias sobre la disposición y la capacidad para aprender matemáticas.

Como se aprecia en la tabla II, el porcentaje de futuros maestros que manifiesta que le gustan las matemáticas tiende a ser ligeramente superior a los que no les gustan (46.1\% frente a un 36.1\%). La variable que marca la diferencia, como se verá más adelante, es el tipo de estudios previos realizados.

Tabla II. Creencias sobre la disposición y la capacidad para aprender matemáticas

\begin{tabular}{l|c|c|c|c|c|c|c}
\hline & 1 & 2 & 3 & 4 & 5 & Media & DT \\
\hline 1. Me gustan las matemáticas. & 25 & 26 & 25 & 34 & 31 & 3.1 & 1.4 \\
2. No todos los estudiantes tienen la & $17.7 \%$ & $18.4 \%$ & $17.7 \%$ & $24.1 \%$ & $22 \%$ & 3.4 & \multirow{2}{*}{0.9} \\
misma capacidad para resolver problemas. & $0.7 \%$ & $5 \%$ & $13.5 \%$ & $24.1 \%$ & $56.7 \%$ & 4.3 & 0.9 \\
3. A algunas personas se les dan mejor las & 7 & 15 & 35 & 40 & 44 & 3.7 & 1.2 \\
matemáticas que a otras. & $5 \%$ & $10.6 \%$ & $24.8 \%$ & $28.4 \%$ & $31.2 \%$ & 3.7 & \multirow{2}{*}{0.5} \\
4. Los estudiantes que son buenos en & 90 & 39 & 9 & 3 & 0 & 1.5 & 0.7 \\
matemáticas son más inteligentes. & $63.8 \%$ & $27.7 \%$ & $6.4 \%$ & $2.1 \%$ & $0 \%$ & \\
\hline
\end{tabular}

Por otro lado, el $80.8 \%$ de los participantes consideran que existen diferentes capacidades para resolver problemas de matemáticas y el $59.6 \%$ creen que hay personas a las que se les dan mejor las matemáticas que a otras. Una primera lectura de estos resultados sugiere que la mayoría de los futuros maestros consideran que la capacidad intelectual es un factor que ejerce un papel importante en el aprendizaje de las matemáticas, lo cual ya ha sido puesto de manifiesto en la literatura previa. Nuestros resultados, sin embargo, complementan los datos previos ya que el $91.5 \%$ de los participantes considera que ser bueno en matemáticas no implica necesariamente ser más inteligente.

El segundo grupo de preguntas del Cuestionario I (ver tabla III) recoge datos acerca de las creencias de los futuros maestros como aprendices de matemáticas. Por esta razón, las cuestiones planteadas se refieren a las relaciones que han ido estableciendo con esta disciplina a lo largo de su escolaridad y, de forma más concreta, inciden sobre todo en su aptitud para aprender la disciplina. 
Tabla III. Creencias sobre la propia aptitud para aprender matemáticas

\begin{tabular}{|c|c|c|c|c|c|c|c|}
\hline & 1 & 2 & 3 & 4 & 5 & Media & DT \\
\hline 5. Yo no sirvo para las matemáticas. & $\begin{array}{c}50 \\
35.5 \%\end{array}$ & $\begin{array}{c}34 \\
24.1 \%\end{array}$ & $\begin{array}{c}29 \\
20.6 \%\end{array}$ & $\begin{array}{c}13 \\
9.2 \%\end{array}$ & $\begin{array}{c}15 \\
10.6 \%\end{array}$ & 2.4 & 1.3 \\
\hline $\begin{array}{l}\text { 6. Las matemáticas me han costado más que } \\
\text { a la mayoría de mis compañeros. }\end{array}$ & $\begin{array}{c}39 \\
27.7 \%\end{array}$ & $\begin{array}{c}37 \\
26.2 \%\end{array}$ & $\begin{array}{c}40 \\
28.4 \%\end{array}$ & $\begin{array}{c}20 \\
14.2 \%\end{array}$ & $\begin{array}{l}5 \\
3.5 \%\end{array}$ & 2.4 & 1.1 \\
\hline $\begin{array}{l}\text { 7. Me gusta resolver problemas de } \\
\text { matemáticas. }\end{array}$ & $\begin{array}{c}24 \\
17 \%\end{array}$ & $\begin{array}{c}35 \\
24.8 \%\end{array}$ & $\begin{array}{c}31 \\
22 \%\end{array}$ & $\begin{array}{c}32 \\
22.7 \%\end{array}$ & $\begin{array}{c}19 \\
13.5 \%\end{array}$ & 2.9 & 1.3 \\
\hline $\begin{array}{l}\text { 8. Cuando resuelvo problemas normalmente } \\
\text { dudo de si está bien o mal. }\end{array}$ & $\begin{array}{c}6 \\
4.3 \%\end{array}$ & $\begin{array}{c}16 \\
11.3 \%\end{array}$ & $\begin{array}{c}46 \\
32.6 \%\end{array}$ & $\begin{array}{c}39 \\
27.7 \%\end{array}$ & $\begin{array}{c}34 \\
24.1 \%\end{array}$ & 3.6 & 1.1 \\
\hline $\begin{array}{l}\text { 9. Cuando no me sale un problema no me } \\
\text { rindo y lo intento de nuevo. }\end{array}$ & $\begin{array}{c}3 \\
2.1 \% \\
\end{array}$ & $\begin{array}{l}9 \\
6.4 \% \\
\end{array}$ & $\begin{array}{c}34 \\
24.1 \% \\
\end{array}$ & $\begin{array}{c}64 \\
45.4 \% \\
\end{array}$ & $\begin{array}{c}31 \\
22 \% \\
\end{array}$ & 3.8 & 0.9 \\
\hline
\end{tabular}

Los datos obtenidos son, en términos generales, poco alentadores: casi un $20 \%$ de los participantes en el estudio considera que no sirven para las matemáticas, a pesar de que la media es de 2.4 puntos. Este es un dato preocupante, sobre todo si tenemos en cuenta que en España los estudios y la profesión de maestro de Infantil y de Primaria son generalistas, por lo que cuando accedan a la profesión es muy posible que tengan que enseñar matemáticas.

Otro aspecto preocupante es que aunque más de la mitad de los participantes opina que su capacidad para resolver tareas matemáticas no difiere de la de sus compañeros, el porcentaje de futuros maestros que afirma que le gusta resolver problemas es sólo del $36.2 \%$, y muchos de ellos confirman que tienen dudas cuando los resuelven (51.8\%). Este es otro aspecto que debería ser tratado de forma explícita durante la formación inicial de maestros, sobre todo si tenemos en cuenta que la resolución de problemas es uno de los ejes centrales del aprendizaje y la enseñanza de las matemáticas.

El último grupo de preguntas del Cuestionario I se centra en las creencias de los futuros maestros sobre el funcionamiento de la clase (ver tabla IV).

Tabla IV. Creencias sobre el funcionamiento de la clase

\begin{tabular}{l|c|c|c|c|c|c|c}
\hline & 1 & 2 & 3 & 4 & 5 & Media & DT \\
\hline 10. Me gustaban las clases de & 32 & 28 & 26 & 25 & 30 & 3.0 & 1.5 \\
matemáticas. & $22.7 \%$ & $19.9 \%$ & $18.4 \%$ & $17.7 \%$ & $21.3 \%$ & 3.0 & \\
11. En mi aprendizaje de las matemáticas & 2 & 5 & 20 & 35 & 79 & 4.3 & 0.9 \\
me influyó mucho el profesor que tuve. & $1.4 \%$ & $3.5 \%$ & $14.2 \%$ & $24.8 \%$ & $56 \%$ & & \\
12. En los exámenes de matemáticas lo & 43 & 30 & 21 & 30 & 17 & 2.6 & 1.4 \\
pasaba peor que en los otros exámenes. & $30.5 \%$ & $21.3 \%$ & $14.9 \%$ & $21.3 \%$ & $12.1 \%$ & & \\
13. Normalmente entendía las explica- & 3 & 22 & 43 & 51 & 22 & 3.5 & 1.0 \\
ciones del profesor de matemáticas. & $2.1 \%$ & $15.6 \%$ & $30.5 \%$ & $36.2 \%$ & $15.6 \%$ & 3.5 & \\
14. Alguna vez he tenido miedo que el & 17 & 29 & 30 & 33 & 32 & 3.2 & 1.3 \\
profesor de matemáticas me preguntara. & $12.1 \%$ & $20.6 \%$ & $21.3 \%$ & $23.4 \%$ & $22.7 \%$ & 3.2 \\
\hline
\end{tabular}

El porcentaje de respuestas a la cuestión "me gustaban las clases de matemáticas" es similar entre los que expresan que no les gustaban y los que sí (42.6\% y 39\%, respectivamente), lo que confirma que las relaciones que los estudiantes han establecido con las matemáticas han sido de distinta naturaleza. Uno de los factores que posiblemente ha tenido mayor peso es la influencia de sus profesores, ya que un $80.8 \%$ expresa que les han influido en el aprendizaje de las matemáticas. A pesar de que la formulación de la pregunta del cuestionario no ha permitido especificar si la influencia ha sido positiva o negativa, las respuestas sobre el grado de comprensión de las explicaciones de sus profesores dejan entrever que hay más participantes con una valoración positiva que negativa (51.8 y 17.7\%, respectivamente). En términos generales, pues, los resultados obtenidos confirman los de estudios previos (Caballero et al., 2008; Ministerio de Educación y Cultura, 2012) -que señalan que el maestro ejerce un notable papel en la construcción del sistema de creencias vinculado al aprendizaje de las matemáticas y su enseñanza. 
El análisis de otros aspectos vinculados al funcionamiento de la clase de matemáticas, como por ejemplo los exámenes o las preguntas que el profesor formula en voz alta, ha evidenciado un escaso grado de seguridad y confianza: el 33.4\% expresa que lo pasaban peor en los exámenes de matemáticas que en los de otras asignaturas y casi la mitad ha tenido miedo de que el profesor de matemáticas les preguntara en clase porque se sentían inseguros.

\subsection{Análisis del nivel de confianza para ejercer como futuros maestros}

En este apartado se presentan los resultados obtenidos en el Cuestionario II, en primer lugar se muestran los datos cuantitativos y enseguida se muestran algunas evidencias que justifican su elección.

La tabla $V$ muestra que únicamente el $23 \%$ de los futuros maestros han elegido las matemáticas en primer lugar como asignatura preferida para enseñar, mientras que los que la escogerían en último lugar representan el $45 \%$ del total. El resto ha elegido otras materias.

Tabla V. Asignatura preferida para enseñar

\begin{tabular}{c|c|c}
\hline Matemáticas en primer lugar & Matemáticas en último lugar & Total general \\
\hline $34(23 \%)$ & $64(45 \%)$ & 141 \\
\hline
\end{tabular}

Los argumentos más repetidos entre los que manifiestan que les gustaría ser profesores de matemáticas son que les gusta la materia, se sienten seguros enseñándola, se consideran buenos en matemáticas, piensan que es una asignatura que permite usar una metodología más participativa y dinámica, o bien porque creen que para enseñar matemáticas deben gustar. También en algunos casos han manifestado que les gustaría enseñar matemáticas porque es una asignatura útil.

En contrapartida, los argumentos que más aparecen entre los que escogen las matemáticas en último lugar son la escasa aptitud, el poco interés o bien la inseguridad. Muchos de ellos manifiestan que no sabrían cómo explicar las matemáticas, no se ven capaces, no las dominan, no las entienden, se les dan mal, no tienen conocimientos suficientes, no les gustan, es una materia muy teórica, etc. Las malas experiencias vividas como estudiantes o la metodología son otros factores que condicionan el hecho de escoger las matemáticas en último lugar. Finalmente, otros argumentos que se mencionan con menor frecuencia son la dificultad de la asignatura, el aburrimiento o la inutilidad de los contenidos, haciendo clara alusión a las matemáticas que les enseñaron durante su trayectoria escolar. Como ya se ha señalado, este es un aspecto preocupante, ya que cuando accedan a un lugar de trabajo es muy posible que tengan que enseñar matemáticas, por lo que es imprescindible que durante la formación inicial se transforme esta visión negativa.

Finalmente, en la tabla VI se presentan los datos referentes a la asignatura con la que los futuros maestros se sienten más seguros para enseñar.

Tabla VI. Grado de seguridad en relación con la enseñanza de las matemáticas

\begin{tabular}{c|c|c}
\hline Sentimiento de seguridad & Sentimiento de inseguridad & Total general \\
\hline $25(18 \%)$ & $42(30 \%)$ & 141 \\
\hline
\end{tabular}

Menos de la mitad de los 141 participantes se han referido a las matemáticas ( $n=67$ ). Los argumentos más repetidos por los participantes con un sentimiento de seguridad hacia las matemáticas (18\%) son la confianza que les ha dado impartir clases particulares de matemáticas, el interés por la materia o bien porque opinan que el nivel de los contenidos de matemáticas de Educación Infantil y Primaria es bajo, pero si tuvieran que enseñar matemáticas en Educación Secundaria Obligatoria (ESO) o Bachillerato ya no se sentirían tan seguros.

La aptitud es uno de los argumentos que aparece con mayor frecuencia entre los estudiantes que mencionan las matemáticas como asignatura con la que se sienten menos seguros (más de la mitad se 
han referido a esta variable). Algunos participantes también han hecho alusión el bajo interés por la materia, la experiencia como alumnos o bien la dificultad de las matemáticas.

Рara comprender con más exactitud cómo influyen las relaciones que los estudiantes han establecido con las matemáticas a lo largo de sus estudios pre-universitarios se ha analizado, mediante la prueba Anova, si los estudios de procedencia ejercen alguna influencia en la disposición hacia las matemáticas.

En la tabla VII se observa que la media más alta corresponde a los participantes que proceden de un bachillerato científico. En cambio, los futuros maestros que proceden del bachillerato humanístico -y sobre todo los que proceden de FP/CFGS- son los que obtienen una media más baja. Рara poder comparar las medias se ha realizado una prueba de comparaciones múltiples usando el método de Tukey: entre los estudiantes procedentes de bachillerato científico y los procedentes de CFGS se ha obtenido un $p$-valor de 0.009 (<0.05), por lo que la diferencia entre ambos grupos es estadísticamente significativa.

Tabla VII. Medias sobre el gusto hacia las matemáticas según el acceso a la universidad

\begin{tabular}{|c|c|c|c|c|c|c|c|c|}
\hline \multirow[b]{2}{*}{ Estudios previos } & \multirow{2}{*}{$\mathrm{N}$} & \multirow{2}{*}{ Media } & \multirow[b]{2}{*}{ DT } & \multirow{2}{*}{$\begin{array}{l}\text { Error } \\
\text { típico }\end{array}$} & \multicolumn{2}{|c|}{$\begin{array}{l}\text { Intervalo de confianza } \\
\text { para la media al 95\% }\end{array}$} & \multirow{2}{*}{ Mín. } & \multirow{2}{*}{ Máx. } \\
\hline & & & & & $\begin{array}{l}\text { Límite } \\
\text { inferior }\end{array}$ & $\begin{array}{l}\text { Límite } \\
\text { superior }\end{array}$ & & \\
\hline Bachillerato científico & 33 & 3.73 & 1.353 & .235 & 3.25 & 4.21 & 1 & 5 \\
\hline Bachillerato humanístico & 44 & 3.09 & 1.444 & .218 & 2.65 & 3.53 & 1 & 5 \\
\hline Licenciados & 4 & 3.50 & 1.291 & .645 & 1.45 & 5.55 & 2 & 5 \\
\hline FP/CFGS & 44 & 2.66 & 1.311 & .198 & 2.26 & 3.06 & 1 & 5 \\
\hline Otros & 16 & 3.31 & 1.448 & .362 & 2.54 & 4.08 & 1 & 5 \\
\hline Total & 141 & 3.14 & 1.417 & .119 & 2.91 & 3.38 & 1 & 5 \\
\hline
\end{tabular}

También se consideró oportuno analizar la relación entre el gusto hacia las matemáticas y la disposición y la seguridad para enseñarlas. Los resultados obtenidos mediante las pruebas Chi-Cuadrado se muestran en las tablas VIII a XI.

Tabla VIII. Tabla de contingencia entre las variables "me gustan las matemáticas" y "la asignatura preferida para enseñar"

\begin{tabular}{c|c|c|c|c|c|c}
\hline \multirow{2}{*}{$\begin{array}{c}\text { Matemáticas } \\
\text { en primer lugar }\end{array}$} & \multicolumn{7}{|c}{ Me gustan las matemáticas } \\
\cline { 2 - 7 } & 1 & 2 & 3 & 4 & 5 & Total \\
\hline \multirow{2}{*}{ No } & 24 & 26 & 22 & 30 & 5 & 107 \\
& $22.4 \%$ & $24.3 \%$ & $20.6 \%$ & $28 \%$ & $4.7 \%$ & $100 \%$ \\
\hline \multirow{2}{*}{ Sí } & 1 & 0 & 3 & 4 & 26 & 34 \\
& $2.9 \%$ & $.0 \%$ & $8.8 \%$ & $11.8 \%$ & $76.5 \%$ & $100 \%$ \\
\hline \multirow{2}{*}{ Total } & 25 & 26 & 25 & 34 & 31 & 141 \\
& $17.7 \%$ & $18.4 \%$ & $17.7 \%$ & $24.1 \%$ & $22 \%$ & $100 \%$ \\
\hline
\end{tabular}

Tabla IX. Prueba de Chi-Cuadrado para la tabla VIII

\begin{tabular}{l|c|c|c}
\hline & Valor & gl & $\begin{array}{c}\text { Sig. asintótica } \\
\text { (bilateral) }\end{array}$ \\
\hline Chi-cuadrado de Pearson & 79,122 & 4 & .000 \\
Razón de verosimilitudes & 77,007 & 4 & .000 \\
N de casos válidos & 141 & & \\
\hline
\end{tabular}

Se ha obtenido un valor del $0.000<0.05$ por lo tanto podemos rechazar la hipótesis nula de que las dos variables son independientes, confirmándose que existe una dependencia entre la disposición hacia las matemáticas y la elección de las matemáticas como asignatura preferida. 
Tabla X. Tabla de contingencia entre las variables "me gustan las matemáticas" y el "grado de seguridad en relación a la enseñanza de las matemáticas"

\begin{tabular}{c|c|c|c|c|c|c}
\hline Matemáticas & \multicolumn{6}{|c}{ Me gustan las matemáticas } \\
\cline { 2 - 7 } más seguro & 1 & 2 & 3 & 4 & 5 & Total \\
\hline \multirow{2}{*}{ No } & 25 & 26 & 23 & 28 & 14 & 116 \\
& $21.6 \%$ & $22.4 \%$ & $19.8 \%$ & $24.1 \%$ & $12.1 \%$ & $100 \%$ \\
Sí & 0 & 0 & 2 & 6 & 17 & 25 \\
& $.0 \%$ & $.0 \%$ & $8.0 \%$ & $24.0 \%$ & $68.0 \%$ & $100 \%$ \\
Total & 25 & 26 & 25 & 34 & 31 & 141 \\
& $17.7 \%$ & $18.4 \%$ & $17.7 \%$ & $24.1 \%$ & $22 \%$ & $100 \%$ \\
\hline
\end{tabular}

Tabla XI. Prueba de Chi-Cuadrado para la tabla X

\begin{tabular}{l|c|c|c}
\hline & Valor & gl & $\begin{array}{c}\text { Sig. asintótica } \\
\text { (bilateral) }\end{array}$ \\
\hline Razón de verosimilitudes & 41.879 & 4 & .000 \\
N de casos válidos & 141 & & .000 \\
\hline
\end{tabular}

En este caso, dado que el p-valor es 0.000 , se puede afirmar que existe una relación entre ambas variables.

\section{Discusión y conclusiones}

En este estudio se han analizado las creencias de los futuros maestros sobre las matemáticas y su enseñanza en general, y la disposición y la aptitud para enseñarlas en particular. Los datos obtenidos acerca de la disposición de los futuros maestros hacia las matemáticas muestran una dispersión importante entre los que indican que les gustan las matemáticas y los que no, confirmándose de esta manera que han establecido diferentes relaciones con dicha asignatura a lo largo de su escolaridad, en la línea ya señalada por Beauchamp y Thomas (2009). Opinan, además, que el rendimiento en matemáticas depende de una capacidad natural. Estos resultados ya se mencionaron en los estudios de Caballero et al. (2008) y del Ministerio de Educación, Cultura y Deporte (2012), entre otros. Sin embargo, un elemento distintivo de la presente investigación es que los futuros maestros no consideran que los mejores alumnos sean los más inteligentes.

Respecto a las creencias de los futuros maestros sobre la propia aptitud para aprender matemáticas, los datos obtenidos confirman un sentimiento de inferioridad ante esta materia (Caballero et al., 2008; Ministerio de Educación, Cultura y Deporte, 2012; López y Alsina, 2016; Op 't Eynde et al., 2002). Un dato novedoso que aporta nuestro estudio es que esta visión difiere de forma estadísticamente significativa según los estudios de procedencia: los participantes que proceden de una formación humanística o de un CFGS subrayan dificultades en los exámenes, inseguridad en las tareas o, en términos más generales, una menor confianza en sus habilidades matemáticas que los que proceden de un bachillerato científico.

Las creencias sobre el funcionamiento de la clase de las matemáticas confirman que el maestro ejerce un papel importante en la visión acerca del aprendizaje de las matemáticas y su enseñanza (Caballero et al., 2008), y han revelado, además, un escaso grado de seguridad y confianza frente a las tareas matemáticas.

Además de las creencias anteriores, se ha indagado también en el nivel de confianza y el sentimiento de seguridad que presentan los futuros maestros para enseñar matemáticas. Los resultados son preocupantes, ya que el $45 \%$ de los participantes señaló que la asignatura que menos prefieren enseñar es matemáticas.

En conclusión, pues, los principales sentimientos negativos que se identificaron en el estudio son la escasa disposición y aptitud hacia las matemáticas y su enseñanza. Como se ha indicado, un grupo 
importante de los participantes manifestó que no sabrían cómo explicar las matemáticas.

Consideramos que, en lugar de que los formadores se queden anclados en la idea de que hay estudiantes para maestro con una visión negativa, durante la formación inicial deberían desarrollar ajustes a esta situación teniendo en cuenta el indiscutible valor social que tienen las matemáticas. En este sentido, coincidimos con Contreras, Penalva y Torregrosa (2011), en que el punto de partida para realizar estos ajustes es impulsar la reflexión de los futuros maestros sobre su propia experiencia formativa con relación a las matemáticas, y también acerca de sus propias expectativas sobre el éxito para que aprendan a tratar la competencia percibida (Di Martino y Zan, 2010).

Así, por ejemplo, para evitar estigmatizar a los alumnos con base en su capacidad, se debería fomentar una visión inclusiva de las matemáticas usando estrategias didácticas como, por ejemplo, la presentación de estudios de caso o el uso de episodios de video y el role playing para ir configurando un perfil docente que contribuya al principio de igualdad en educación matemática, es decir, que las matemáticas pueden y deben ser aprendidas por todos (NCTM, 2000). Asimismo, para que no reproduzcan modelos de profesor inadecuados, una labor imprescindible durante la formación inicial debería consistir en favorecer la toma de conciencia de estos modelos de profesor y ofrecer alternativas de actuación, en la línea señalada por Kaasila et al. (2012).

De forma más general, y considerando los sentimientos negativos identificados, sería preciso usar metodologías de enseñanza fundamentadas en la reflexión sistemática que permitan deconstruir estos sentimientos y, a la vez, co-construir nuevos conocimientos científicos y profesionales. Algunas de las estrategias didácticas que deberían aplicar los formadores y los aspectos que deberían tratar en el marco de estas metodologías reflexivas son los siguientes (Alsina, Batllori, Falgàs y Vidal, 2019):

Experiencias previas: situaciones pasadas que han dejado una huella negativa en la construcción de la identidad matemática o en la construcción de la identidad profesional para enseñar matemáticas.

Creencias sobre uno mismo: la mirada interna como aprendiz o como futuro docente de matemáticas.

Creencias sobre el funcionamiento de la clase: las concepciones acerca de las líneas metodológicas de los centros escolares, la forma de gestionar las prácticas de enseñanza e incluso los distintos tipos de relaciones entre los profesionales.

Conocimientos disciplinares implícitos: la visión acerca de las matemáticas y los conocimientos que las integran.

Conocimientos didácticos implícitos: la visión acerca de la forma de enseñar matemáticas.

Interacción con el contexto I (centro escolar): el contraste con el contexto del aula de infantil o de primaria a partir de la observación participante y del aprendizaje situado, principalmente.

Interacción con el contexto II (la universidad): el contraste con el contexto pedagógico (en este caso, en la universidad) a partir también de la participación activa y el aprendizaje situado.

Interacción con uno mismo: el diálogo interno mediante la introspección.

Interacción entre iguales: la comunicación con los demás, a través del trabajo cooperativo, la negociación y el diálogo reflexivo.

Interacción con el experto: la comunicación con el más experto, también a través del diálogo reflexivo y la negociación.

Interacción con la teoría: el contraste con el conocimiento tanto disciplinar como didáctico vinculado con la construcción del perfil docente para enseñar matemáticas. 
Durante la aplicación de estos aspectos es posible, como señalan Gellert et al. (2013), que surjan resistencias y subversiones si los aprendices para maestro consideran que el cambio es imposible o no deseado. Por esta razón, una formación inicial de los maestros de matemáticas que conduzca al cambio debería proporcionar conocimiento disciplinar y didáctico que permita transformar los sentimientos negativos detectados a través de metodologías reflexivas, con el objeto de resolver la problemática planteada. En futuros estudios será necesario aplicar las estrategias didácticas mencionadas para analizar su impacto en el proceso de construcción autorregulado del propio perfil docente de los futuros maestros, que incluye tanto conocimiento profesional como pensamiento crítico.

\section{Referencias}

Alsina, Á., Batllori, R., Falgàs, M., y Vidal, I. (2019). Marcas de autorregulación para la construcción del perfil docente durante la formación de maestros. Revista Complutense de Educación, 30(1), 55-74.

Beauchamp, C. y Thomas, L. (2009). Understanding teacher identity: an overview of issues in the literature and implications for teacher education. Cambridge Journal of Education, 39(2), 175-189.

Caballero, A., Blanco, L. J., y Guerrero, E. (2008). El dominio afectivo en futuros maestros de matemáticas en la Universidad de Extremadura. Paradigma, XXIX(2), 157-171.

Contreras, P., Penalva, M. C., y Torregrosa, G. (2011). Identidad profesional y conocimiento matemático para la enseñanza de maestros en formación. En M. Marín, G. Fernández, L. J. Blanco y M. Palarea (Eds.), Investigación en Educación Matemática XV (pp. 329-338). Ciudad Real: SEIEM.

DeBellis, V. A. y Goldin, G. A. (1999). Aspects of affect: Mathematical intimacy, mathematical integrity. En O. Zaslovsky (Ed.), Actas de la 23 Conferencia International Group for the Psychology of Mathematics Education (Vol. 2, pp. 249-256). Haifa, Israel: PME.

Di Martino, P. y Zan, R. (2010). "Me and maths": towards a definition of attitude grounded on students' narratives. Journal of Mathematics Teacher Education, 13(1), pp. 27-48.

Gellert, U., Espinoza, L., y Barbé, J. (2013). Being a mathematics teacher in times of reform. zDM-The International Journal on Mathematics Education, 45(4), 535-545.

Gómez-Chacón, I. M. (2000). Matemática emocional. Los afectos en el aprendizaje matemático. Madrid: Narcea.

Gómez-Chacón, I. M., Op 't Eynde, P., y De Corte, E. (2006). Creencias de los estudiantes de matemáticas. La influencia del contexto de clase. Enseñanza de las Ciencias, 24(3), 309-324.

Hannula, M.S., Op 't Eynde, P., Schlöglmann, W., y Wedege, T. (2007). Affect and mathematical thinking. En D. Pitta-Pantazi y G. Philippou (Eds.), European Research in Mathematics Education V; Proocedings of the Fifht Congress of The European Society for Research in Mathematics Education (pp. 202-208). Nicosia: University of Cyprus.

Kaasila, R., Hannula, M. S., y Laine, A. (2012). "My personal relationship towards mathematics has necessarily not changed but..." Analyzing pre-service teachers' mathematical identity talk. International Journal of Science and Mathematics Education, 10, 975-995.

López, P. y Alsina, A. (2013). ¿Cómo influyen las creencias sobre uno mismo en relación a las matemáticas? Una aproximación al papel de la identidad matemática en futuros maestros. En A. Berciano, G. Gutiérrez, A. Estepa y N. Climent (Eds.), Investigación en Educación Matemática XVII (pp. 345-353). Bilbao: SEIEM. 
López, P. y Alsina, A. (2016). Creencias de los futuros maestros sobre la aptitud matemática: consideraciones para promover procesos de cambio en la formación inicial. Bolema, 30(56), 892-905.

McLeod, D. B. (1992). Research on affect in mathematics education: a reconceptualization. En D. A. Grouws (Ed.), Handbook of research on mathematics learning and teaching (pp. 575-596). Nueva York: McMillan.

Ministerio de Educación, Cultura y Deporte (2012). TEDS-M. Informe español. Estudio internacional sobre la formación inicial en matemáticas de los maestros. Madrid: Subdirección General de Documentación y Publicaciones.

National Council of Teachers of Mathematics. (2000). Principles and standards for school mathematics. Reston, vA: NCTM.

National Council of Teachers of Mathematics. (2015). De los principios a la acción: para garantizar el éxito matemático de todos. Reston, vA: NCTM.

Op 't Eynde, P., De Corte, E. y Verschaffel, L. (2002). "Framing students' mathematics-related beliefs: a quest for conceptual clarity and a comprehensive categorizacion". En G. Leder, E. Pehkonen y G. Torner (Eds.), Beliefs: a hidden variable in mathematics education? (pp. 13-37). Dordrecht, Países Bajos: Kluwer Academic Publishers. 